\title{
Exceptionally low blood glucose response to dried beans: comparison with other carbohydrate foods
}

\author{
DAVID J A JENKINS, THOMAS M S WOLEVER, \\ RODNEY H TAYLOR, HELEN M BARKER, \\ HASHMEIN FIELDEN
}

\section{Summary and conclusions}

Normal volunteers took 50-g carbohydrate portions of eight varieties of dried legumes and 24 common foods drawn from grains, cereals and pasta, breakfast cereals, biscuits, and tuberous vegetables. Both the mean peak rise in blood glucose concentration and mean area under the glucose curve of the subjects who ate beans were at least $45 \%$ lower than those of subjects who ate the other foods.

These results suggest a potentially valuable role for dried leguminous seeds in carbohydrate exchanges for individuals with impaired carbohydrate tolerance.

\section{Introduction}

Not all carbohydrate sources raise the blood glucose to the same extent when fed in equivalent amounts. Such factors as food form,,$^{1-3}$ the nature of the carbohydrate, ${ }^{4}$ and the dietary fibre content ${ }^{5}$ may in part explain these differences.

Recent work on the dietary control of diabetics ${ }^{6-8}$ has focused attention on these factors. There is now evidence that high-fibre, high-carbohydrate diets may greatly improve control and reduce urinary glucose loss. ${ }^{9}$ At the same time, these diets and others which result in flatter glucose and insulin responses ${ }^{10}$ have been found to lower the fasting triglyceride concentration in hypertriglyceridaemic individuals. ${ }^{11}$

Preliminary studies suggest that leguminous seeds are one of the high-fibre, high-carbohydrate foods which cause only a small rise in the blood glucose. ${ }^{12}$ We therefore compared them with other more common high-carbohydrate foods to see whether these may usefully be exchanged for beans in the diets of diabetics and hypertriglyceridaemic individuals.

\section{Subjects and methods}

After overnight fasts groups of five to 10 healthy volunteers $(15 \mathrm{men}$, 10 women; $110+3 \%$ ideal body weight) took test meals containing a 50-g carbohydrate portion of a single food. These included cooked beans and peas, cereal grains, breads and pasta, breakfast cereals, biscuits, and tuberous vegetables. Altogether 35 foods were tested. The portion sizes were calculated from food tables. ${ }^{13} 14$

Dry grains, legumes, and vegetables were cooked by boiling in a minimum of water with $2 \mathrm{~g}$ salt. Breakfast cereals were taken with $300 \mathrm{ml}$ milk. To increase palatability, all meals included at least $250 \mathrm{ml}$ of tea made with one tea bag, $50 \mathrm{ml}$ of milk, and water so that the final meal volume was over $600 \mathrm{ml} .120 \mathrm{~g}$ of skinned, pipped tomato was

\footnotetext{
Department of the Regius Professor of Medicine, Radcliffe Infirmary, Oxford

DAVID J A JENKINS, DM, research associate

University Laboratory of Physiology, Oxford

THOMAS M S WOLEVER, MSC, research fellow

Department of Gastroenterology, Central Middlesex Hospital, London NW10

RODNEY H TAYLOR, MRCP, Wellcome senior research fellow HELEN M BARKER, BSC, SRD, research dietitian HASHMEIN FIELDEN, SRN, research sister
}

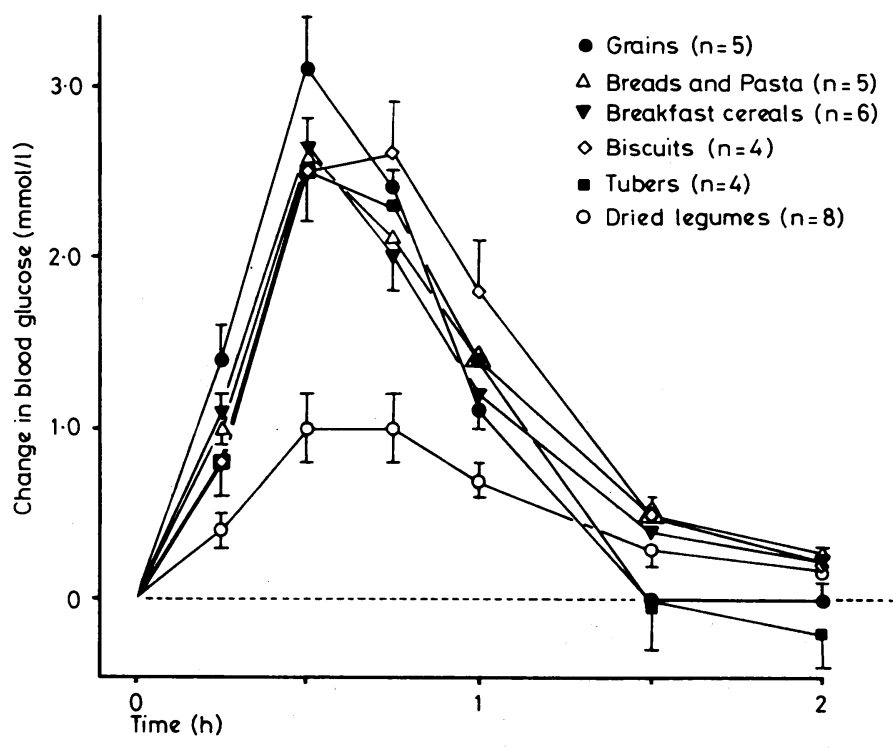

Change in blood glucose concentration after eating $50-\mathrm{g}$ carbohydrate portions of individual grains, breads and pasta, breakfast cereals, biscuits, tubers, and dried legumes.

Conversion: SI to traditional units-Glucose: $1 \mathrm{mmol} / 1 \approx 18 \mathrm{mg} / 100 \mathrm{ml}$.

added to the spaghetti, rice, bread, millet, buckwheat, and legumes.

To ensure that the groups selected to test each food were drawn from a similar population with respect to glucose tolerance, at least one standard 50-g glucose tolerance test was performed on each subject and more where subjects tested more than one food-that is, one test for every two to three foods. Tests were performed over the same time as the respective meals in $550 \mathrm{ml}$ of tea with $50 \mathrm{ml}$ of milk (except for the cereal test, where $250 \mathrm{ml}$ of tea and $350 \mathrm{ml}$ of milk were used).

All test meals were eaten in the morning over 10 or 15 minutes after 10- to 12-hour overnight fast and standard physical activity and meal times on the previous day. Finger prick samples were obtained with Autolet lancets $0,15,30,45,60,90$, and 120 minutes after the start of the meal. Hands were warmed between electric blankets to ensure good blood flow. Blood samples were collected into tubes containing $83 \mu \mathrm{g}$ of sodium fluoride and $250 \mu \mathrm{g}$ of potassium oxalate and stored on crushed ice or frozen at $-20^{\circ} \mathrm{C}$ before analysis of glucose by a glucose oxidase method (Yellow Springs Instruments, 23AM Glucose Analyser). ${ }^{15}$

Areas under the two-hour glucose curve were calculated, and the results are given as means \pm standard error of the mean (SEM). The significance of the difference between foods was calculated using Student's $t$ test for unpaired data assuming unknown variances to be equal.

\section{Results}

The mean blood glucose response after the eight dried leguminous seeds lay significantly below the mean curves of all the other food groups (see figure) on at least two occasions over the two-hour test period. Both the mean peak rise in glucose concentration and area under the glucose curve were also significantly lower after the dried legumes. The mean glucose area and peak rise after beans were, respectively, only $51 \%(\mathrm{p}<0.001)$ and $41 \%(\mathrm{p}<0.001)$ of the values after the grains; $51 \%(\mathrm{p}<0.01)$ and $45 \%(\mathrm{p}<0.001)$ of those after the bread and spaghetti; 45\% (p<0.001) and 43\% (p<0.001) of those after the biscuits; $51 \%(\mathrm{p}<0.001)$ and $48 \%(\mathrm{p}<0.001)$ of those after 
Mean blood glucose concentrations, glucose areas, peak rises, and areas of glucose tolerance test (GTT) for groups of 5-10 subjects who took one or more carbohydrate food

\begin{tabular}{|c|c|c|c|c|c|c|c|c|c|c|c|c|}
\hline & & \multirow{2}{*}{$\begin{array}{c}\text { No } \\
\text { of } \\
\text { subjects }\end{array}$} & \multicolumn{7}{|c|}{ Blood glucose $(\mathrm{mmol} / \mathrm{l})$ at time $(\mathrm{min})$ : } & \multirow{2}{*}{$\begin{array}{c}\text { Mean } \\
\text { - glucose area } \\
(\text { mmol min/l) }\end{array}$} & \multirow{2}{*}{$\begin{array}{c}\text { Mean } \\
\text { peak rise } \\
(\mathrm{mmol} / \mathrm{l})\end{array}$} & \multirow{2}{*}{$\begin{array}{c}\text { Mean area } \\
\text { of GTT } \\
(\mathrm{mmol} \mathrm{min} / 1\end{array}$} \\
\hline & & & 0 & 15 & 30 & 45 & 60 & 90 & 120 & & & \\
\hline \multicolumn{13}{|c|}{ Dried legumes } \\
\hline $\begin{array}{l}\text { Beans: } \\
\text { Butter beans } \\
\text { Haricot beans } \\
\text { Kidney beans } \\
\text { Soya beans } \\
\text { Blackeye peas } \\
\text { Chick peas } \\
\text { Marrowfat peas } \\
\text { Lentils } \quad . \\
\end{array}$ & $\begin{array}{l}. \\
\because \\
\because \\
\therefore \\
\therefore \\
\cdots \\
\therefore\end{array}$ & $\begin{array}{l}6 \\
6 \\
7 \\
5 \\
6 \\
6 \\
6 \\
7 \\
\end{array}$ & $\begin{array}{l:l}4 \cdot 2 & 0 \cdot 2 \\
4 \cdot 4 & 0 \cdot 1 \\
4 \cdot 3 & 0 \cdot 2 \\
4 \cdot 3 & 0 \cdot 1 \\
4 \cdot 2 & 0 \cdot 1 \\
4 \cdot 3 & 0 \cdot 2 \\
4 \cdot 2 & 0 \cdot 1 \\
4 \cdot 3 & 0 \cdot 2 \\
\end{array}$ & $\begin{array}{ll}4 \cdot 7 & 0 \cdot 2 \\
4 \cdot 6 & 0 \cdot 1 \\
4 \cdot 5 & 0 \cdot 2 \\
4 \cdot 6 & 0 \cdot 1 \\
4 \cdot 6 & 0 \cdot 1 \\
4 \cdot 7 & 0 \cdot 2 \\
5 \cdot 1 & 0 \cdot 2 \\
4 \cdot 8 & 0 \cdot 2 \\
\end{array}$ & $\begin{array}{l}5 \cdot 6: 0 \cdot 2 \\
5 \cdot 1: 0 \cdot 2 \\
4 \cdot 8=0 \cdot 1 \\
4 \cdot 6: 0 \cdot 1 \\
5 \cdot 1: 0 \cdot 1 \\
5 \cdot 5: 0 \cdot 2 \\
6 \cdot 6: 0 \cdot 2 \\
5 \cdot 2=0 \cdot 2 \\
\end{array}$ & $\begin{array}{l}5 \cdot 5=0 \cdot 3 \\
5 \cdot 5=0 \cdot 2 \\
4 \cdot 9=0 \cdot 2 \\
4 \cdot 4=0 \cdot 2 \\
5 \cdot 2=0 \cdot 1 \\
5 \cdot 6=0 \cdot 3 \\
6 \cdot 1=0 \cdot 3 \\
5 \cdot 0=0 \cdot 2 \\
\end{array}$ & $\begin{array}{l}5 \cdot 1: 0 \cdot 4 \\
5 \cdot 5 \pm 0 \cdot 3 \\
4 \cdot 9=0 \cdot 3 \\
4 \cdot 3=0 \cdot 1 \\
5 \cdot 2: 0 \cdot 2 \\
5 \cdot 3=0 \cdot 4 \\
4 \cdot 9=0 \cdot 3 \\
4 \cdot 8=0 \cdot 2 \\
\end{array}$ & $\begin{array}{l}4 \cdot 6: 0.3 \\
4 \cdot 8 \pm 0 \cdot 3 \\
5 \cdot 0: 0 \cdot 3 \\
4 \cdot 1: 0 \cdot 1 \\
4 \cdot 8 \pm 0 \cdot 2 \\
4 \cdot 7 \pm 0 \cdot 4 \\
4 \cdot 1=0 \cdot 2 \\
4 \cdot 7: 0 \cdot 2 \\
\end{array}$ & $\begin{array}{l}4 \cdot 6=0 \cdot 2 \\
4 \cdot 5=0 \cdot 2 \\
4 \cdot 7=0 \cdot 2 \\
4 \cdot 1=0 \cdot 1 \\
4 \cdot 5=0 \cdot 1 \\
4 \cdot 8=0 \cdot 3 \\
4 \cdot 2=0 \cdot 1 \\
4 \cdot 9=0 \cdot 2 \\
\end{array}$ & $\begin{array}{ll:}84 \cdot 7 & 12 \cdot 6 \\
72 \cdot 8 & 16 \cdot 1 \\
64 \cdot 3 & 17 \cdot 7 \\
16 \cdot 8 & 6 \cdot 1 \\
74 \cdot 0 & 11 \cdot 2 \\
81 \cdot 7 & 16 \cdot 3 \\
98 \cdot 5 & 8 \cdot 3 \\
59 \cdot 7 & 6 \cdot 1 \\
\end{array}$ & $\begin{array}{l}1 \cdot 6 \pm 0 \cdot 1 \\
1 \cdot 2=0.3 \\
1 \cdot 0 \pm 0 \cdot 2 \\
0 \cdot 4=0 \cdot 1 \\
1 \cdot 2 \pm 0 \cdot 1 \\
1 \cdot 4 \pm 0 \cdot 3 \\
2 \cdot 5 \pm 0 \cdot 2 \\
0.9 \pm 0 \cdot 1\end{array}$ & $\begin{array}{l}232 \cdot 5 \pm 14 \cdot 6 \\
223 \cdot 3 \pm 20 \cdot 3 \\
228 \cdot 3 \pm 13 \cdot 1 \\
188 \cdot 0 \pm 18 \cdot 2 \\
230 \cdot 3 \pm 23 \cdot 4 \\
221 \cdot 5 \pm 19 \cdot 9 \\
212 \cdot 3 \pm 18 \cdot 0 \\
212 \cdot 9 \pm 20 \cdot 9 \\
\end{array}$ \\
\hline Mean & .. & & $4 \cdot 3: 0.03$ & $4 \cdot 7=0 \cdot 1$ & $5 \cdot 3=0 \cdot 2$ & $5 \cdot 3 \pm 0 \cdot 2$ & $5 \cdot 0 \pm 0 \cdot 1$ & $4 \cdot 6 \pm 0 \cdot 1$ & $4 \cdot 5 \pm 0 \cdot 1$ & $69 \cdot 1 \pm 8 \cdot 6$ & $1 \cdot 3 \pm 0 \cdot 2$ & $218 \cdot 6 \pm 5 \cdot 1$ \\
\hline \multicolumn{13}{|c|}{ Root vegetables } \\
\hline $\begin{array}{l}\text { Instant mash } \\
\text { New potato } \ldots \\
\text { Sweet potato... } \\
\text { Yams } \quad . . \\
\end{array}$ & $\begin{array}{l}\cdots \\
\cdots \\
\cdots \\
\end{array}$ & $\begin{array}{l}8 \\
8 \\
5 \\
5 \\
\end{array}$ & $\begin{array}{l}4 \cdot 8 \div 0 \cdot 1 \\
4 \cdot 5: 0 \cdot 1 \\
4 \cdot 3 \div 0 \cdot 3 \\
4 \cdot 2 \div 0 \cdot 2 \\
\end{array}$ & $\begin{array}{l}5 \cdot 5=0 \cdot 2 \\
5 \cdot 2=0 \cdot 2 \\
5 \cdot 4=0 \cdot 4 \\
5 \cdot 2=0 \cdot 3 \\
\end{array}$ & $\begin{array}{l}7.0=0.3 \\
7.5=0.4 \\
6.9=0.2 \\
6.5=0.2 \\
\end{array}$ & $\begin{array}{l}7 \cdot 1=0 \cdot 3 \\
8 \cdot 0=0 \cdot 5 \\
6 \cdot 2=0 \cdot 3 \\
6 \cdot 0=0 \cdot 4 \\
\end{array}$ & $\begin{array}{l}6 \cdot 6=0.5 \\
6.9=0.2 \\
4.9=0.6 \\
5 \cdot 0=0.4\end{array}$ & $\begin{array}{l}5 \cdot 2=0 \cdot 4 \\
4 \cdot 3: 0 \cdot 1 \\
4 \cdot 5 \pm 0 \cdot 4 \\
4 \cdot 0 \pm 0 \cdot 2 \\
\end{array}$ & $\begin{array}{l}4 \cdot 8 \pm 0 \cdot 3 \\
4 \cdot 1 \pm 0 \cdot 1 \\
4 \cdot 2 \pm 0 \cdot 3 \\
4 \cdot 2 \pm 0 \cdot 1 \\
\end{array}$ & $\begin{array}{l}138 \cdot 3 \pm 18 \cdot 3 \\
155 \cdot 8+18 \cdot 8 \\
103 \cdot 0=6.9 \\
104 \cdot 2 \pm 25 \cdot 8\end{array}$ & $\begin{array}{l}2.8 \pm 0.3 \\
3.5 \pm 0.5 \\
2.7 \pm 0.3 . \\
2.5 \pm 0.4\end{array}$ & $\begin{array}{l}193 \cdot 6 \pm 27 \cdot 2 \\
228 \cdot 6 \pm 17 \cdot 0 \\
226 \cdot 0 \pm 24 \cdot 7 \\
204 \cdot 8 \pm 27 \cdot 7 \\
\end{array}$ \\
\hline Mean & $\ldots$ & & $4 \cdot 5 \div 0 \cdot 1$ & $5 \cdot 3=0 \cdot 1$ & $7 \cdot 0 \pm 0 \cdot 2$ & $6 \cdot 8 \pm 0.5$ & $5.9 \pm 0.5$ & $4 \cdot 5 \pm 0 \cdot 3$ & $4 \cdot 3 \pm 0 \cdot 2$ & $125 \cdot 3 \pm 13 \cdot 0$ & $2 \cdot 9 \pm 0 \cdot 2$ & $213 \cdot 3 \pm 8 \cdot 4$ \\
\hline \multicolumn{13}{|c|}{ Cereals and cereal products } \\
\hline $\begin{array}{l}\text { Buckwheat } \\
\text { Millet } \\
\text { Rice (brown) } \\
\text { Rice (white) } \\
\text { Sweetcorn } \\
\end{array}$ & $\begin{array}{l}\cdots \\
\cdots \\
\cdots \\
\cdots \\
\end{array}$ & $\begin{array}{l}5 \\
5 \\
7 \\
7 \\
5 \\
\end{array}$ & $\begin{array}{l:c}4 \cdot 3 & 0 \cdot 1 \\
4 \cdot 3 & 0 \cdot 2 \\
4 \cdot 2 & 0 \cdot 1 \\
4 \cdot 1 & 0 \cdot 1 \\
4 \cdot 7 & =0 \cdot 2 \\
\end{array}$ & $\begin{array}{l}5 \cdot 2=0.2 \\
5 \cdot 8=0.3 \\
5 \cdot 7=0.3 \\
5 \cdot 4=0.3 \\
6.4=0.3 \\
\end{array}$ & $\begin{array}{l}6.5=0.5 \\
7.9=0.3 \\
7.6=0.3 \\
7.0=0.3 \\
7.8=0.3 \\
\end{array}$ & 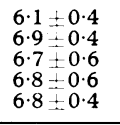 & $\begin{array}{l}5 \cdot 2=0 \cdot 2 \\
5 \cdot 4=0 \cdot 2 \\
5 \cdot 4=0 \cdot 4 \\
5 \cdot 7 \approx 0.6 \\
5 \cdot 2=0.4 \\
\end{array}$ & $\begin{array}{l}4 \cdot 3=0 \cdot 2 \\
4 \cdot 0=0 \cdot 3 \\
4 \cdot 3=0 \cdot 1 \\
4 \cdot 8 \pm 0 \cdot 3 \\
3 \cdot 9=0 \cdot 2 \\
\end{array}$ & $\begin{array}{l}4 \cdot 4 \pm 0 \cdot 1 \\
4 \cdot 1 \pm 0 \cdot 2 \\
4 \cdot 3 \pm 0 \cdot 03 \\
4 \cdot 4 \pm 0 \cdot 3 \\
4 \cdot 2=0 \cdot 2 \\
\end{array}$ & $\begin{array}{r}99 \cdot 8=15 \cdot 8 \\
140 \cdot 8 \pm 14 \cdot 2 \\
150 \cdot 4 \pm 17 \cdot 9 \\
166.9 \pm 29 \cdot 1 \\
118 \cdot 0 \pm 14.8 \\
\end{array}$ & $\begin{array}{l}2 \cdot 4 \pm 0 \cdot 4 \\
3 \cdot 6 \pm 0 \cdot 3 \\
3 \cdot 5 \pm 0 \cdot 2 \\
3 \cdot 3 \pm 0 \cdot 3 \\
3 \cdot 2 \pm 0.4\end{array}$ & $\begin{array}{l}207 \cdot 4 \pm 25 \cdot 3 \\
207 \cdot 4 \pm 25.3 \\
227 \cdot 9 \pm 24.2 \\
227 \cdot 9 \pm 24 \cdot 2 \\
209 \cdot 8 \pm 18 \cdot 8 \\
\end{array}$ \\
\hline Mean & .. & & $4 \cdot 3 \pm 0 \cdot 1$ & $5 \cdot 7 \pm 0 \cdot 2$ & $7 \cdot 4=0.3$ & $6 \cdot 7 \pm 0 \cdot 1$ & $5 \cdot 4 \pm 0 \cdot 1$ & $4 \cdot 3 \div 0 \cdot 2$ & $4 \cdot 3 \pm 0 \cdot 1$ & $135 \cdot 2 \pm 11 \cdot 9$ & $3 \cdot 2 \pm 0 \cdot 2$ & $216 \cdot 1 \pm 4 \cdot 9$ \\
\hline \multirow{2}{*}{\multicolumn{3}{|c|}{ 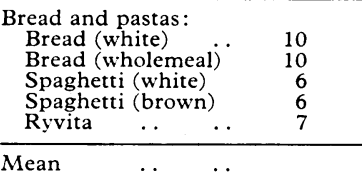 }} & 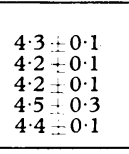 & $\begin{array}{l}5 \cdot 1: 0 \cdot 2 \\
5 \cdot 1: 0.2 \\
5 \cdot 1=0.3 \\
5 \cdot 8=0.3 \\
5 \cdot 3=0 \cdot 1 \\
\end{array}$ & $\begin{array}{l}7 \cdot 1 \pm 0 \cdot 3 \\
7 \cdot 1=0 \cdot 3 \\
6 \cdot 3=0 \cdot 2 \\
6 \cdot 9 \pm 0 \cdot 2 \\
7 \cdot 1 \pm 0 \cdot 3 \\
\end{array}$ & $\begin{array}{l:l}7 \cdot 2 & 0.4 \\
6 \cdot 8 & 0.5 \\
5 \cdot 5 & 0.4 \\
5 \cdot 3 & 0.6 \\
7 \cdot 4 & 0.3 \\
\end{array}$ & 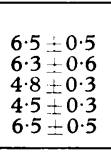 & $\begin{array}{l}4 \cdot 7: 0 \cdot 2 \\
4 \cdot 9: 0 \cdot 3 \\
4 \cdot 6: 0 \cdot 1 \\
4 \cdot 4: 0 \cdot 2 \\
5 \cdot 2: 0 \cdot 3 \\
\end{array}$ & $\begin{array}{l}4 \cdot 3 \div 0 \cdot 2 \\
4 \cdot 2 \pm 0 \cdot 2 \\
4 \cdot 6 \div 0 \cdot 1 \\
4 \cdot 6 \pm 0 \cdot 2 \\
5 \cdot 0: 0 \cdot 2 \\
\end{array}$ & $\begin{array}{r}162.5 \div 19 \cdot 1 \\
173 \cdot 4 \div 23.7 \\
95 \cdot 5 \div 13.9 \\
78 \cdot 3 \pm 7.0 \\
173.4 \pm 27 \cdot 7 \\
\end{array}$ & $\begin{array}{l}3 \cdot 3 \pm 0.3 \\
3 \cdot 4 \pm 0.3 \\
2 \cdot 2 \pm 0.2 \\
2 \cdot 4 \pm 0.1 \\
3 \cdot 1 \pm 0.4 \\
\end{array}$ & $\begin{array}{l}237 \cdot 3 \pm 23 \cdot 9 \\
237 \cdot 3 \pm 23 \cdot 9 \\
192 \cdot 8 \pm 13 \cdot 8 \\
192 \cdot 8 \pm 13 \cdot 8 \\
250 \cdot 3 \pm 10 \cdot 2 \\
\end{array}$ \\
\hline & & & $4 \cdot 3 \pm 0 \cdot 1$ & $5 \cdot 3 \div 0 \cdot 1$ & $6 \cdot 9 \pm 0 \cdot 2$ & $6 \cdot 4 \pm 0 \cdot 4$ & $5 \cdot 7 \pm 0 \cdot 4$ & $4 \cdot 8 \pm 0 \cdot 1$ & $4 \cdot 5 \pm 0 \cdot 1$ & $136 \cdot 6 \pm 20 \cdot 6$ & $2 \cdot 9 \pm 0.2$ & $222 \cdot 1 \pm 12 \cdot 2$ \\
\hline $\begin{array}{l}\text { Breakfast cereals: } \\
\text { All-Bran ... } \\
\text { Cornflakes . } \\
\text { Muesli ... } \\
\text { Porridge oats } \\
\text { Shredded Wheat } \\
\text { Weetabix ... } \\
\end{array}$ & $\begin{array}{l}\cdots \\
\cdots \\
\cdots \\
\cdots \\
\therefore\end{array}$ & $\begin{array}{l}6 \\
6 \\
6 \\
6 \\
6 \\
6\end{array}$ & $\begin{array}{l}4 \cdot 3+0 \cdot 2 \\
4 \cdot 3=0 \cdot 2 \\
4 \cdot 3=0 \cdot 2 \\
4 \cdot 7 \div 0 \cdot 4 \\
4 \cdot 3=0 \cdot 2 \\
4 \cdot 3=0 \cdot 2\end{array}$ & $\begin{array}{l}5 \cdot 6 \pm 0.4 \\
5 \cdot 7 \pm 0.3 \\
5 \cdot 5 \pm 0.3 \\
5 \cdot 6 \pm 0 \cdot 4 \\
5 \cdot 2 \pm 0.3 \\
5 \cdot 6 \pm 0 \cdot 2 \\
\end{array}$ & $\begin{array}{l}6 \cdot 4 \pm 0 \cdot 1 \\
7 \cdot 4 \pm 0.5 \\
7 \cdot 2 \pm 0 \cdot 4 \\
6 \cdot 9 \pm 0.5 \\
6 \cdot 8 \pm 0.5 \\
7 \cdot 0 \pm 0 \cdot 4 \\
\end{array}$ & $\begin{array}{l}5 \cdot 6 \pm 0.3 \\
7 \cdot 0=0.5 \\
6 \cdot 6 \pm 0.5 \\
6 \cdot 2 \pm 0.5 \\
6 \cdot 4 \pm 0.6 \\
6 \cdot 6 \pm 0.5 \\
\end{array}$ & $\begin{array}{l}4 \cdot 8 \pm 0.3 \\
5 \cdot 9=0.7 \\
5 \cdot 5=0.4 \\
5 \cdot 7=0.6 \\
5 \cdot 8=0.7 \\
5 \cdot 7 \pm 0.5 \\
\end{array}$ & $\begin{array}{l}4 \cdot 7 \pm 0 \cdot 2 \\
4 \cdot 9 \pm 0 \cdot 4 \\
4 \cdot 6=0 \cdot 2 \\
4 \cdot 8 \pm 0 \cdot 5 \\
4 \cdot 8 \pm 0 \cdot 4 \\
5 \cdot 0 \pm 0 \cdot 4\end{array}$ & $\begin{array}{l}4 \cdot 8 \div 0 \cdot 2 \\
4 \cdot 5=0.3 \\
4 \cdot 5=0.2 \\
4 \cdot 6=0.4 \\
4 \cdot 7=0.3 \\
4 \cdot 7=0.3 \\
\end{array}$ & $\begin{array}{c}102 \cdot 5 \pm 9 \cdot 9 \\
171 \cdot 2: 25 \cdot 8 \\
136 \cdot 5 \pm 22 \cdot 2 \\
98 \cdot 0 \pm 17 \cdot 7 \\
145 \cdot 0 \pm 30 \cdot 4 \\
156 \cdot 7 \pm 25 \cdot 0 \\
\end{array}$ & $\begin{array}{l}2 \cdot 3 \pm 0 \cdot 1 \\
3 \cdot 2 \pm 0 \cdot 3 \\
2 \cdot 9 \pm 0 \cdot 3 \\
2 \cdot 3 \pm 0 \cdot 4 \\
2 \cdot 8 \pm 0 \cdot 4 \\
2 \cdot 8 \pm 0 \cdot 3 \\
\end{array}$ & $\begin{array}{l}208 \cdot 2 \pm 22 \cdot 3 \\
208 \cdot 2 \pm 22 \cdot 3 \\
208 \cdot 2 \pm 22 \cdot 3 \\
208 \cdot 2 \pm 22.3 \\
208 \cdot 2 \pm 22 \cdot 3 \\
208 \cdot 2 \pm 22.3 \\
\end{array}$ \\
\hline Mean & .. & & $4 \cdot 4 \div 0 \cdot 1$ & $5 \cdot 5 \pm 0 \cdot 1$ & $7 \cdot 0 \pm 0 \cdot 1$ & $6 \cdot 4 \pm 0 \cdot 2$ & $5 \cdot 6 \pm 0 \cdot 2$ & $4 \cdot 8 \pm 0 \cdot 1$ & $4 \cdot 6 \pm 0 \cdot 1$ & $135 \cdot 0 \pm 12 \cdot 0$ & $2 \cdot 7 \pm 0 \cdot 1$ & $208 \cdot 2 \pm 0$ \\
\hline $\begin{array}{ll}\text { Biscuits: } & \\
\text { Digestive } & . . \\
\text { Oatmeal } & \ldots \\
\text { Rich Tea } & \cdots \\
\text { Water } & . \\
\end{array}$ & $\begin{array}{l}. \\
\cdots \\
\cdots \\
\end{array}$ & $\begin{array}{l}6 \\
6 \\
6 \\
6 \\
\end{array}$ & $\begin{array}{l}4 \cdot 4=0 \cdot 2 \\
4 \cdot 5=0 \cdot 2 \\
4 \cdot 8=0 \cdot 2 \\
4 \cdot 6=0 \cdot 2\end{array}$ & $\begin{array}{l}5 \cdot 3 \pm 0.3 \\
5 \cdot 7 \pm 0.2 \\
5 \cdot 7 \pm 0.2 \\
5 \cdot 0 \pm 0.2\end{array}$ & $\begin{array}{l}6 \cdot 5=0.3 \\
7 \cdot 1=0.2 \\
7 \cdot 7=0.2 \\
7 \cdot 0=0.4 \\
\end{array}$ & $\begin{array}{l}6.7=0.3 \\
6.8 \pm 0.5 \\
7.6 \pm 0.5 \\
7.7 \pm 0.4\end{array}$ & $\begin{array}{l}6 \cdot 0 \pm 0.3 \\
6 \cdot 0 \pm 0.5 \\
6 \cdot 4 \pm 0.5 \\
7 \cdot 3 \pm 0.6 \\
\end{array}$ & $\begin{array}{l}5 \cdot 2 \pm 0 \cdot 1 \\
5 \cdot 1 \pm 0 \cdot 2 \\
4 \cdot 8 \pm 0 \cdot 4 \\
5 \cdot 4 \pm 0 \cdot 6 \\
\end{array}$ & $\begin{array}{l}4 \cdot 8 \pm 0 \cdot 1 \\
4 \cdot 8 \pm 0 \cdot 2 \\
4 \cdot 8 \div 0 \cdot 1 \\
4 \cdot 7 \pm 0 \cdot 3\end{array}$ & $\begin{array}{l}144 \cdot 8 \pm 17 \cdot 6 \\
149 \cdot 5 \pm 12 \cdot 4 \\
145 \cdot 2 \pm 12 \cdot 2 \\
177 \cdot 5 \pm 23 \cdot 7 \\
\end{array}$ & $\begin{array}{l}2 \cdot 5 \pm 0.2 \\
2 \cdot 8 \pm 0 \cdot 2 \\
3 \cdot 2 \pm 0.2 \\
3 \cdot 4 \pm 0.3\end{array}$ & $\begin{array}{l}248 \cdot 7 \pm 9 \cdot 8 \\
282 \cdot 0 \pm 32 \cdot 6 \\
274 \cdot 2 \pm 33 \cdot 1 \\
288 \cdot 2 \pm 30 \cdot 1 \\
\end{array}$ \\
\hline Mean & . & & $4 \cdot 6 \div 0 \cdot 1$ & $5 \cdot 4 \div 0 \cdot 2$ & $7 \cdot 1=0 \cdot 3$ & $7 \cdot 2=0 \cdot 3$ & $6 \cdot 4 \pm 0 \cdot 3$ & $5 \cdot 1 \pm 0 \cdot 1$ & $4 \cdot 8 \pm 0.03$ & $154 \cdot 3 \pm 7 \cdot 8$ & $3 \cdot 0 \pm 0 \cdot 2$ & $273 \cdot 3 \pm 8 \cdot 7$ \\
\hline \multicolumn{2}{|c|}{$\begin{array}{l}\text { Mean for all cereals and } \\
\text { cereal products }\end{array}$} & 20 & $4 \cdot 4 \div 0.04$ & $5 \cdot 5 \pm 0 \cdot 1$ & $7 \cdot 1 \pm 0 \cdot 1$ & $6 \cdot 7 \pm 0 \cdot 1$ & $5 \cdot 7 \pm 0 \cdot 2$ & $4 \cdot 7 \pm 0 \cdot 1$ & $4 \cdot 6 \pm 0 \cdot 1$ & $139 \cdot 3 \pm 6 \cdot 8$ & $2 \cdot 9 \pm 0 \cdot 1$ & $226 \cdot 7 \pm 6 \cdot 4$ \\
\hline \multicolumn{13}{|c|}{ Other carbohydrate foods } \\
\hline $\begin{array}{l}\text { Fresh peas } \\
\text { Heinz Baked Beans } \\
\text { Tinned soya beans }\end{array}$ & $\begin{array}{l}\cdots \\
\cdots \\
\cdots\end{array}$ & $\begin{array}{l}6 \\
7 \\
6\end{array}$ & $\begin{array}{l}4 \cdot 2=0 \cdot 1 \\
4 \cdot 5=0 \cdot 1 \\
4 \cdot 4=0 \cdot 1\end{array}$ & $\begin{array}{l}5 \cdot 2 \pm 0 \cdot 2 \\
5 \cdot 4 \pm 0 \cdot 2 \\
4 \cdot 7 \pm 0 \cdot 2\end{array}$ & $\begin{array}{l}6 \cdot 9 \pm 0.3 \\
6 \cdot 6 \pm 0 \cdot 1 \\
5 \cdot 0 \pm 0 \cdot 1\end{array}$ & $\begin{array}{l}5 \cdot 9=0 \cdot 4 \\
6 \cdot 6 \pm 0.5 \\
4 \cdot 7 \pm 0 \cdot 3\end{array}$ & $\begin{array}{l}4 \cdot 7 \pm 0 \cdot 3 \\
5 \cdot 2 \pm 0 \cdot 4 \\
4 \cdot 6 \pm 0 \cdot 2\end{array}$ & $\begin{array}{l}4 \cdot 2 \pm 0 \cdot 1 \\
4 \cdot 3 \pm 0 \cdot 1 \\
4 \cdot 4 \pm 0 \cdot 2\end{array}$ & $\begin{array}{l}4 \cdot 5: 0 \cdot 1 \\
4 \cdot 5: 0 \cdot 1 \\
4 \cdot 5 \pm 0 \cdot 2\end{array}$ & $\begin{array}{l}96 \cdot 3 \pm 15 \cdot 0 \\
92 \cdot 1 \pm 11 \cdot 0 \\
30 \cdot 0 \pm 6 \cdot 9\end{array}$ & $\begin{array}{l}2 \cdot 7 \pm 0 \cdot 3 \\
2 \cdot 3 \pm 0 \cdot 2 \\
0 \cdot 7 \pm 0 \cdot 1\end{array}$ & $\begin{array}{l}213 \cdot 0 \pm 17 \cdot 9 \\
233 \cdot 7 \pm 20 \cdot 1 \\
215 \cdot 2 \pm 19 \cdot 5\end{array}$ \\
\hline
\end{tabular}

Conversion: SI to traditional units-Glucose: $1 \mathrm{mmol} / 1 \approx 18 \mathrm{mg} / 100 \mathrm{ml}$.

the breakfast cereals; and $55^{\circ},(\mathrm{p}<0.01)$ and $45^{\circ}(\mathrm{p}<0.001)$ of those after the tubers.

Most of the leguminous seeds were similar in their effect on blood glucose, with glucose areas of $60-85 \mathrm{mmol} \mathrm{min} / 1$ (1081-1531 $\mathrm{mg}$ $\mathrm{min} / 100 \mathrm{ml}$ ). The two exceptions were the high response to marrowfat peas of $99 \mathrm{mmol} \mathrm{min} / 1(1784 \mathrm{mg} \mathrm{min} / 100 \mathrm{ml})$ and the very low one of $17 \mathrm{mmol} \mathrm{min} / 1$ (306 $\mathrm{mg} \mathrm{min} / 100 \mathrm{ml}$ ) to soya beans (see table).

Group means of the other foods all fell between 125 and 154 $\mathrm{mmol} \mathrm{min} / 1$ (2252 and $2775 \mathrm{mg} \mathrm{min} / 100 \mathrm{ml}$ ), and no individual mean for any food lay more than $30 \%$ away from its group mean, most being within $20^{\circ}{ }_{0}^{\circ}$ of the group mean.

Fresh frozen garden peas produced a relatively large peak rise and glucose area, similar to those of the dried peas but different from those of the other leguminous seeds tested. The canned baked beans in tomato sauce showed a $92^{\circ},(\mathrm{p}<0.01)$ greater peak rise than the plain haricot beans, although the canned soya beans, also in tomato sauce, were not significantly different from the plain soya beans.

Except when eating biscuits, the mean glucose areas for the glucose tolerance tests taken by each group of subjects who tested a food were all similar, and the comparison of glucose areas of the different foods was therefore considered justified. The mean age of the subjects testing biscuits was older than that of the other groups, and the mean glucose area on the glucose tolerance test was significantly higher than the test glucose areas of the other food groups (by $27^{\circ} \%$ ). The biscuit values therefore appeared artificially high. Nevertheless, the mean glucose area of the group who ate the beans, which was only $45 \%$ of that of the group who ate the biscuits, was still only $56 \%$ of the biscuits group's value when a correction was made for the $25 \%$ difference in areas on the glucose tolerance test of the two groups.

\section{Discussion}

With the possible exception of dried peas, beans gave very much flatter blood glucose responses than other foods containing the same amount of carbohydrate.

Leguminous seeds are rich sources of dietary fibre ${ }^{13}$ and may also contain starch in a form that is more resistant to enzymic breakdown ${ }^{12}$ than that in more commonly consumed foods. Both these factors have been suggested as important in governing the rate of release of carbohydrate from the gastrointestinal tract $^{45}$ and thus determining the suitability of foods for inclusion in the diabetic diet.

The leguminous seed fibre guar gum has been used successfully in the diet to reduce urinary glucose loss in diabetics, ${ }^{7}$ and a high-fibre, high-carbohydrate diet containing generous daily portions of beans, as judged by their contribution to total fibre intake ${ }^{6}$ allowed insulin to be withdrawn in most diabetics taking 
less than 30 units/day. Perhaps even more surprisingly, such diets have successfully reduced serum triglyceride concentrations in hypertriglyceridaemic men, ${ }^{11}$ even though diets high in carbohydrate from conventional sources are reported to cause hypertriglyceridaemia. ${ }^{16}$ Nevertheless, such diets may require the consumption of relatively large amounts of leguminous seed carbohydrate because no reduction in serum triglyceride concentrations was seen when only $6 \%$ of the calories were derived from dried legumes. ${ }^{17}$

Meals of soya beans and lentils have also been shown to raise the blood glucose concentrations of diabetics only $30 \%$ of that seen when meals containing an equal amount of carbohydrate as wholemeal bread were taken. ${ }^{12}$ Since three of the diabetics described were insulin dependent, it is unlikely that the effect was due to increased insulin secretion, since their responses were similar to those of the others with $\beta$-cell function. Previous studies using added dietary fibre ${ }^{18}$ or looking at the glucose response to carbohydrate-rich foods ${ }^{2}$ have noted that when the blood glucose response is reduced more than expected even larger reductions are generally seen in the serum insulin response.

Compared with bread, leguminous seed starches are digested more slowly in vitro by human digestive juices. ${ }^{12}$ Intraluminal starch digestion has not previously been considered to be ratelimiting on the basis of the excess amount of amylase present. ${ }^{19}$ Nevertheless, no account has been taken of difference in fibre content ${ }^{5}$ and the type of starch $^{4}$ found in different foods, and it may be through modification of such gastrointestinal functions as transit, digestion, and absorption that these factors may have an effect.

The dramatic effect of beans as a class on postprandial glycaemia has not hitherto been reported because legumes have been neglected in the limited comparative testing of individual foods on blood glucose. ${ }^{420}$ For the foods that have been tested our own results are in broad agreement with the findings of others, ${ }^{40}$ except for potato, which in one study ${ }^{20}$ was considered to produce only half the rise seen with bread.

From the epidemiological point of view, there is an additional reason why it may be desirable to reintroduce leguminous seeds in the Western diet. Recently, it has been claimed that dietary fibre is important in preventing the development of many of the diseases of Western civilisation (colon cancer, diverticular disease, coronary arterial disease, etc). ${ }^{21}$ Yet the epidemiological leads on which this is based would do equally well for leguminous seeds, which form so much of a part of the diet in rural Africa, India, Asia, and Latin America and are so conspicuously absent from the modern industrial Western diet.

The leguminous dishes that are still eaten in the West include peas and canned baked beans in a sugar-rich tomato sauce. Both produce higher postprandial rises in blood glucose concentration than other legumes. Both fresh garden peas and mature marrowfat peas were similar, although the nature of the carbohydrate would have been different, with more starch and less sugar in the mature seed. Peas may therefore be different from beans. Nevertheless, the replacement of over $45 \%$ of the carbohydrate as sugar in the baked beans abolished some of the reduction predicted from the results with plain haricot beans.

We conclude from our results of testing individual foods that leguminous seeds as a class produce by far the lowest rise in postprandial blood glucose of the carbohydrate-rich foods tested. It has already been shown that a meal of soya beans and lentils produces very little increase in the blood glucose concentration compared with an equivalent amount of carbohydrate as wholemeal bread. ${ }^{12}$ Legumes may perhaps be incorporated usefully into the diabetic diet to increase the carbohydrate intake and so reduce fat consumption. Substitution for other forms of carbohydrate in the diet of the hypertriglyceridaemic individual may also aid the reduction of fasting serum triglyceride concentrations. This would be especially useful at a time when reports of side effects have made certain drug treatments less attractive. ${ }^{22}$
We thank Mr Peter Lees and Mr David Heath of Speywood Laboratories for providing the oatmeal biscuits; and are grateful for the great help of Mr Mike Hogan, who together with Mr Bill Williams, Mr Nick Daw, Mr Richard Studerest, and Mr Andrew Lawrence took part in testing the foods. Canned soya beans were kindly provided by the Granose Food Company, Watford, Herts.

The work was supported by grants from the British Diabetic Association and the Medical Research Council to DJAJ.

\section{References}

1 Douglas JM. Raw diet and insulin requirements. Ann Intern Med 1975 ; 82:61.

${ }^{2}$ Haber GB, Heaton KW, Murphy D. Depletion and disruption of dietary fibre: effects on satiety, plasma-glucose and serum insulin. Lancet 1977; ii :679-82.

${ }^{3}$ Leeds AR, Gassull MA, Metz GL, Jenkins DJA. Food: influence of form on absorption. Lancet 1975 ;ii:1213.

${ }^{4}$ Crapo PA, Reaven G, Olefsky J. Postprandial plasma-glucose and insulin responses to different complex carbohydrates. Diabetes $1977 ; 26: 1178$.

5 Jenkins DJA, Wolever TMS, Leeds AR, et al. Dietary fibres, fibre analogues and glucose tolerance: importance of viscosity. $\mathrm{Br} \mathrm{Med} \mathcal{F}$ $1978 ; i: 1392-4$.

${ }^{6}$ Anderson JW, Ward K. High-carbohydrate, high-fiber diets for insulintreated men with diabetes mellitus. Am $\mathcal{F}$ Clin Nutr 1979;32:2312-21.

7 Jenkins DJA, Wolever TMS, Nineham R, et al. Guar crispbread in the diabetic diet. $B r M e d \mathcal{F} 1978$;ii:1744-6.

${ }^{8}$ Miranda PM, Horwitz DL. High fibre diets in the treatment of diabetes mellitus. Ann Intern Med 1978;88:482-6.

9 Anderson JW, Midgley WR, Wedman B. Fiber and diabetes. Diabetes Care $1979 ; 2: 369-79$.

${ }^{10}$ Albrink MJ, Newman T, Davidson PC. Effect of high- and low-fiber diets on plasma lipids and insulin. Am f Clin Nutr 1979;32:1486-91.

${ }^{11}$ Anderson JW. Triglyceride lowering effects of high fiber diets. Am $\mathcal{F}$ Clin Nutr 1979;32:933.

12 Jenkins DJA, Wolever TMS, Taylor RH, et al. Rate of digestion of foods and postprandial glycaemia in normal and diabetic subjects. $\mathrm{Br} \mathrm{Med} \mathrm{F}$ 1980 ;281:14-17.

13 Paul AA, Southgate DAT. McCance and Widdowson's the composition of foods. 4th ed. London: HMSO, 1978. (Medical Research Council Special Report Series No 297.)

${ }^{14}$ Platt BS. Tables of representative values of foods commonly used in tropical countries. London: HMSO, 1962. (Medical Research Council Special Report Series No 302.)

${ }^{15}$ Clark LC Jr. A polarographic enzyme electrode for the measurement of oxidase substrates. In: Kessler M, Bruley DF, Leland CC, Lübbers DW, Silver IA, Strauss J, eds. Oxygen supply. Munich: Urban and Schwarzenberg, 1973: 120-8.

${ }^{16}$ Glueck CJ, Levy R, Fredrickson DS. Immunoreactive insulin, glucose tolerance and carbohydrate inducibility of types II, III, IV and V hyperlipoproteinaemia. Diabetes 1969;18:739.

17 Grande F, Anderson JT, Keys A. Sucrose and various carbohydratecontaining foods and serum lipids in man. Am $\mathcal{F}$ Clin Nutr 1974 ;27: 1043-51.

${ }^{18}$ Jenkins DJA, Leeds AR, Gassull MA, Cochet B, Alberti KGMM. Decrease in postprandial insulin and glucose concentrations by guar and pectin. Ann Intern Med 1977;86:20-3.

19 Dahlqvist A, Borgstrom B. Digestion and absorption of disaccharides in man. Biochem $\mathcal{F} 1961 ; 81: 411-8$.

20 Schauberger G, Brinck UC, Suldner G, Spaethe R, Niklas L, Otto H. Exchange of carbohydrates according to their effect on blood glucose. Diabetes $1977 ; 26: 415$.

${ }^{21}$ Burkitt DP, Trowell HC, eds. Refined carbohydrate foods and disease. New York: Academic Press, 1975.

${ }^{22}$ Report from the Committee of Principal Investigators. A co-operative trial in the primary prevention of ischaemic heart disease using clofibrate. Br Heart f 1978;40:1067-118.

(Accepted 14 fuly 1980)

ONE HUNDRED YEARS AGO The New York Medical Record records two cases in which the new anaesthetic bromide of ethyl was administered. It required eleven minutes to bring one patient under its influence, and nine drachms were used. Its odour was very disagreeable, its effect was evanescent. Two drachms had previously put the house-physician asleep, and, during its administration to the patient, the doctor-who appears to be unusually susceptible to the effects of anaesthetics-came near falling asleep. Another patient, a very strong man, just as he was coming under the influence of the same anaesthetic, became excited, violent, and unmanageable, sprang from the table, and escaped to his bed. This is not a very satisfactory "record," but it probably does not represent the results of very skilled administration. (British Medical fournal, 1880.) 